\title{
Systematic identification and treatment of smokers by hospital based cessation practitioners in a secondary care setting: cluster randomised controlled trial
}

\author{
(c) $(1)$ (8)
}

\author{
$\mathrm{R} \mathrm{L}$ Murray lecturer in health policy and promotion ${ }^{1}$, J Leonardi-Bee associate professor of medical \\ statistics ${ }^{1}$, J Marsh research assistant ${ }^{1}$, L Jayes research assistant ${ }^{1}$, J Li research fellow ${ }^{2}$, S Parrott \\ senior research fellow ${ }^{2}$, J Britton professor of epidemiology ${ }^{1}$
}

${ }^{1}$ Division of Epidemiology and Public Health and UK Centre for Tobacco and Alcohol Studies, University of Nottingham, Nottingham City Hospital, Nottingham NG5 1PB, UK; ' 2 Department of Health Sciences and UK Centre for Tobacco and Alcohol Studies, University of York, Heslington, York, UK

\begin{abstract}
Objectives To investigate the effectiveness of the systematic default provision of smoking cessation support to all adult smokers admitted to hospital, relative to usual care.

Design Open, cluster randomised controlled trial.

Setting Acute medical wards in one large teaching hospital in the United Kingdom.

Participants 264 patients randomised to intervention and 229 to usual care; primary outcome data were available at four weeks for 260 and 224 patients, respectively. All adult smokers and recent ex-smokers able to give informed consent were eligible for entry into the study.

Interventions The intervention comprised systematic smoking ascertainment and default provision of behavioural support and cessation pharmacotherapy for the duration of the hospital stay for all smokers and recent ex-smokers, with follow-up and referral to community services after discharge. Usual care comprised cessation support delivered at the initiative and discretion of clinical staff. All staff and patients were aware of group assignment.
\end{abstract}

Main outcome measures Smoking cessation at four weeks, validated by measuring exhaled carbon monoxide. Secondary outcomes were uptake of inpatient behavioural support, use of cessation pharmacotherapy, referral to and uptake of community support after discharge, and validated smoking cessation at six months. Participants lost to follow-up were assumed to have reverted to smoking.

Results All patients in the intervention group received at least brief advice to quit smoking, compared to 106 (46\%) patients in the usual care group. Cessation at four weeks was achieved by $38 \%(n=98)$ of intervention patients and $17 \%(n=37)$ of usual care patients (adjusted odds ratio 2.10 (95\% confidence interval 0.96 to 4.61 ), $P=0.06$, number of patients needed to treat 8 ). Uptake of inpatient behavioural support, use of pharmacotherapy, and referral to and uptake of community support after discharge were all substantially and statistically significantly higher in the intervention group than in the usual care group. Cessation at six months was achieved by $19 \%(n=47)$ of intervention and $9 \%(n=19)$ of usual care patients, although this difference was not significant (adjusted odds ratio 1.53 (95\% confidence interval 0.60 to 3.91 ); $P=0.37$ ).

Conclusions Substantial improvements in smoking cessation among smokers admitted to hospital can be achieved by systematic ascertainment and delivery of cessation support in secondary care.

Trial registration International Standard Randomised Controlled Trial Number ISRCTN25441641.

\section{Introduction}

Tobacco smoking is the largest avoidable cause of premature death and disability in both developed and developing countries. Half of all lifelong smokers die as a consequence of their smoking, typically from lung cancer, chronic obstructive pulmonary disease, or cardiovascular disease. ${ }^{1}$ Average life expectancy among smokers is 10 years less than among never smokers, and quitting smoking generates considerable benefits in both quantity and quality of life, almost irrespective of age at quitting. ${ }^{1}$ Interventions to help smokers to quit are effective and highly cost effective, ${ }^{23}$ and should therefore be offered to all smokers as a routine component of healthcare provision. ${ }^{45}$ Admission to hospital is a powerful opportunity to promote smoking cessation, since it imposes at least temporary smoking abstinence at a time when smokers are probably particularly 
aware of the health consequences of smoking and, in many cases, have reduced or quit smoking as a result of the condition precipitating admission. High intensity interventions - defined as advice or behavioural support to increase patients' motivation to quit that is initiated during a hospital stay and including at least one month of support after discharge-have been shown to increase smoking cessation among patients in hospital, regardless of the diagnosis at admission. ${ }^{6}$ Ascertaining smoking status in all patients, and providing cessation support for smokers willing to accept it, has been recommended in clinical guidelines in the United States and United Kingdom for over 15 years. ${ }^{4} 8$ However, implementation of this guidance in secondary care settings remains far from complete. ${ }^{9-12}$

Research into smoking cessation in secondary care so far has tended to focus on the behavioural and pharmacological content of interventions, rather than methods of maximising delivery and uptake. Therefore, we investigated a service designed systematically to identify and offer conventional, evidence based support during smoking cessation ${ }^{4}$ to all smokers admitted to acute medical wards in a UK secondary care setting. This study was conducted to determine whether such a service was effective in increasing validated cessation of smoking and in promoting cessation of service uptake, relative to usual care provision.

\section{Methods}

We used a parallel, cluster randomised design to study all smokers who reported that they were current smokers, or had smoked within four weeks of admission, to any of 18 medical wards at a large teaching hospital in the UK (Nottingham City Hospital, Nottingham). To minimise contamination between intervention and usual care groups, we used admission ward as the unit of randomisation. Patient recruitment started on 11 October 2010 and ended on 9 August 2011. The trial was activated with the National Institute for Health Research in September 2010, and the International Standard Randomised Controlled Trial Number (ISRCTN) issued on 28 October 2010. The delay between the start of patient enrolment and the issue of the ISRCTN arose from an administrative misunderstanding, and the trial was registered as soon as possible after this was discovered.

\section{Randomisation and masking}

Wards (clusters) were allocated by random sequence generation to deliver either intervention or usual care by the Nottingham Clinical Trials Unit. We stratified wards by the number of discharges per week to achieve approximate parity in numbers between groups (a discharge rate of $\leq 10$ patients per week or below was considered to be low; $>10$ patients per week was considered to be high). Patients were then cluster randomised to either intervention or usual care, according to the ward to which they were admitted. Clinical and research staff and patients were aware of group assignment.

\section{Usual care}

For patients on usual care wards, smoking status on admission was ascertained in accordance with hospital standard practice. This practice involved non-obligatory completion of three checkboxes on an admission form by the admitting doctor, indicating whether the patient is a smoker-and if so, how many cigarettes were smoked per day and pack years and whether the patient wanted to receive cessation support. Advice to quit and offers of cessation support were then given to patients at the discretion of and in accordance with the usual practice of doctors and other health professionals involved in their care. Patients in the usual care group were recruited to the trial at the end of their hospital admission, usually on the day of discharge. But patients were recruited up to 48 hours in advance if discharge was planned for a weekend, when a researcher screened the clinical notes of all patients and, if smoking data were incomplete, confirmed smoking status by direct questioning. We ascertained whether patients were smokers, or whether they had smoked in the four weeks before admission to hospital. Smokers were then given written information about the study and asked to provide a measure of exhaled carbon monoxide and consent to be contacted to ascertain smoking status at four weeks and six months after discharge. Written information about the study explained that smoking cessation services in the hospital were being assessed; that depending on the ward of admission, one of two forms of support to help quit smoking would be provided; and the measurements and follow-up that the study entailed (web appendix). Any smoker who requested cessation support at this point was given brief cessation advice by the researcher, and the patient's supervising doctor was informed of the request.

\section{Intervention}

On intervention wards, researchers identified all new admissions on the first weekday morning after admission and ascertained smoking status from the admission form or, if incomplete, by direct questioning. All patients who had smoked within four weeks of admission were given written information about the study. They were also asked to consent to provide a measurement of exhaled carbon monoxide at discharge, and to be contacted four weeks and six months after discharge to ascertain smoking status. The written information given to patients was identical to that used in the usual care group. Patients received details of confidentiality and ethics approval for the research, but no further details of the intervention.

All patients well enough to engage in discussion were given brief advice to quit, and offered help to do so, by the research team. The brief advice included an explanation of the benefits of quitting, the nature of the support available (tailored support, one to one counselling or behavioural support, and pharmacotherapy while in hospital and continued support after discharge), and an assessment of desire to receive support. Patients were provided with standard written information from the UK's health service about smoking cessation, if interested. ${ }^{13}$ Patients who were too ill to communicate were reviewed and engaged when their condition had improved sufficiently to do so.

Those who accepted cessation support were visited at the bedside by one of three smoking cessation practitioners. These practitioners had all received either university or healthcare professional education, and were trained in intensive behavioural support (through the intensive support and advice smoking cessation training delivered by the local NHS stop smoking service). Such training included awareness of smoking demographics, the health effects of smoking and stopping smoking, smoking cessation treatments and their outcome, motivational interviewing, and behavioural support techniques. The smoking cessation practitioners offered one to one counselling, to be delivered daily throughout admission (or as often as was acceptable to the patient). In the absence of contraindications, they prescribed dual nicotine replacement therapy comprising a 16 hour, $21 \mathrm{mg}$ transdermal patch combined with a fast acting product (chosen from gum, lozenge, inhalator, or nasal spray) according to preference. If dual nicotine replacement therapy was contraindicated or if the patient preferred bupropion or varenicline treatment, the 
supervising doctor was asked to advise and prescribe as appropriate.

One to one counselling was based on standard sessions of behavioural support from the local stop smoking service. During these sessions, the smoking cessation practitioners used motivational interviewing techniques to encourage a quit attempt with advice tailored according to the admission diagnosis and personal circumstances, and explained choices regarding dual nicotine replacement therapy and its use. In line with standard protocol for the local stop smoking service, the first consultation would typically last between 20 and 30 minutes, with subsequent sessions lasting up to 10 minutes. Patients who declined support after receiving brief advice were given standard NHS written information on quitting smoking. Patients with cardiac disorders were also given a British Heart Foundation booklet about stopping smoking. ${ }^{14}$ On discharge, all participants were offered referral to a local stop smoking service for further cessation support, and contacted by telephone by the smoking cessation practitioners at least once. Participants were also contacted at four weeks and six months to ascertain smoking status and, if abstinent, to arrange verification based on measuring levels of exhaled carbon monoxide.

Most doctors responsible for patients on the study wards agreed that all patients under their care could be approached to take part in the study, although in some cases, they asked that particular individuals be excluded on clinical grounds. Doctors responsible for oncology care initially declined permission for the study team to approach any of their patients, on the grounds that focusing on smoking could cause distress. After negotiation, it was agreed that any patient on an oncology ward with an active or in-date order in the medical notes with "do not resuscitate" should not be approached, and that for all other patients on the oncology ward, consent was to be specifically sought from the supervising doctor before approaching the patient.

\section{Outcome measures}

The primary outcome was four weeks of smoking cessation, defined as self reported abstinence for four weeks and validated by measuring a level of exhaled carbon monoxide of $10 \mathrm{ppm} .{ }^{15}$ Secondary outcomes included the proportions (\%) of smokers who were offered and accepted behavioural support or pharmacotherapy as inpatients, were discharged with cessation therapy and with post-discharge support arranged, were given support from a local stop smoking service after hospital discharge, were abstinent from smoking with validation based on measuring levels of exhaled carbon monoxide at discharge, and had self reported continuous abstinence for six months with validation based on measuring levels of exhaled carbon monoxide.

\section{Statistical analysis}

We used Stata 11 MP to compare demographic characteristics between intervention and usual care groups. Comparisons used two sample $t$ tests, Mann-Whitney U tests, or $\chi^{2}$ tests as appropriate; primary and secondary outcomes using a multilevel model including a random effect for clustering at ward level; and the stratification variable (baseline discharge rates) as a fixed covariate. We performed two retrospective sensitivity analyses to assess the robustness of our findings. The first assessed the effect of further adjusting the primary analysis model for sex; the second assessed the effect of excluding oncology care wards from the primary analysis model. Excluding oncology wards avoided the potential for bias arising from the decision by the consultant oncologists to decline consent for most of their patients to be invited to participate in the study. If ward random effects could not be estimated, we used logistic regression with adjustment using robust standard errors. For patients admitted more than once, we included data for the first admission only. All analyses were based on intention to treat, including all patients who consented to participate and assuming relapse to smoking in the absence of validated cessation. Patients who died before discharge, before four week follow-up, or before six month follow-up, were excluded at the respective time points. $\mathrm{P}$ values lower than 0.05 were deemed significant.

The number of patients needed to be treated (NNT) for one additional person to be quit at four weeks (continuous abstinence) was estimated using the event rate in the usual care group and odds ratio from the primary outcome analysis. We calculated the sample size using an expected cessation rate of $13 \%$ in patients receiving usual care (based on data from 2003 in the same setting $\left.{ }^{16}\right)$. Allowing for clustering with an intraclass correlation coefficient of $0.02,500$ smokers (about 28 per ward) were needed to provide $80 \%$ power at $5 \%$ significance for us to detect a doubling of cessation at four weeks to $26 \%$.

\section{Results}

We identified 1072 patients admitted to 18 study wards (that is, clusters) between October 2010 and August 2011, representing about $14 \%$ of all admissions, who had smoked within four weeks of admission. Of these patients, 138 were admitted briefly and discharged before contact could be made, 28 were judged to be unable to give informed consent, and 12 were repeat admissions. Of the remaining 894 patients, 92 (10\%) were excluded by their supervising doctor; most of these exclusions $(n=59)$ were among the 104 eligible patients admitted to oncology wards. Of 802 patients asked to participate, 309 declined consent, and 493 (mean age 56 years (range 18-91), $296(60 \%)$ male) were allocated to treatment groups (264 to intervention, 229 to usual care; fig $\downarrow$ ). Patients in the intervention group were slightly younger and more likely to be male (table $1 \Downarrow$ ). The length of stay of study participants ranged from one to 98 days (median five days); median length of stay was slightly longer in the intervention than the usual care group (six days $v$ five days; $P=0.05$, Mann-Whitney $U$ test). The number of behavioural support sessions delivered in the intervention group ranged from zero to 12 sessions (median one day, interquartile range zero to two days).

Dual nicotine replacement therapy was the most widely used pharmacotherapy (66 (29\%) patients on usual care wards, 133 (50\%) on intervention wards) - most commonly as a transdermal patch, an inhalator, or both. Varenicline was used by only five patients, all on intervention wards, and bupropion was not used by any patient in either group. Ward randomisation resulted in the majority of patients receiving usual care $(56 \%, n=129)$ coming from respiratory wards, and the majority of those receiving the intervention $(57 \%, \mathrm{n}=151)$ coming from cardiac wards. Follow-up measurements at four weeks were conducted between November 2010 and September 2011, and six month follow-up measurements were conducted between April 2011 and February 2012. Analyses for primary and secondary outcomes were conducted on nine clusters in each treatment group in all instances, with the exception of the retrospective analysis, which excluded oncology wards (analysing six clusters in the intervention group and eight clusters in the control group). We attempted to contact all patients at four week and six month follow-up on no fewer than five separate occasions, primarily 
by telephone at different times of day and including one written attempt to collect follow-up data. Smoking status was ascertained in $243(92 \%)$ patients in the intervention group and $192(84 \%)$ in the usual care group at four weeks, and in 195 (74\%) and 155 (68\%) patients at six months, respectively. Patients who were not contactable or who withdrew consent to continue in the study at each follow-up stage were considered to be still smoking and included in the intention to treat analysis.

\section{Primary outcome}

Validated quit rates at four weeks were over twice as high in the intervention (38\%) than usual care group (17\%). With initial adjustment for clustering and stratification by discharge numbers, this effect was not significant at the $5 \%$ level (adjusted odds ratio 2.10 (95\% confidence interval 0.96 to 4.61 ), 18 clusters, intraclass correlation coefficient $0.077, \mathrm{P}=0.06$, $\mathrm{NNT}=8$ ), and a sensitivity analysis further adjusting for the effect of patients' sex had marginal effects on the findings (2.09 ( 0.95 to 4.60$), 18,0.077, \mathrm{P}=0.07$ ). To assess whether the intervention effect was strongly influenced by data from patients on the oncology wards, where consultants declined access to a majority of patients, we carried out a retrospective sensitivity analysis excluding these patients; four week cessation in the remaining 440 patients was $17 \%$ in the usual care and $42 \%$ in the intervention group (2.71 ( 1.33 to 5.54$), 14,0.038, \mathrm{P}=0.006$ ).

\section{Secondary outcomes}

All patients on intervention wards and less than half of those on usual care wards received at least brief advice to quit smoking. Uptake of behavioural cessation support, use of pharmacotherapy, and referral to and uptake of the local stop smoking service were all significantly higher in the intervention group than in the usual care group $(\mathrm{P}<0.001$ in all cases). Validated abstinence from smoking at discharge and at six months was also higher in the intervention group than in the usual care group, but this difference was not significant $(\mathrm{P}=0.37$; table $2 \Downarrow)$. An economic analysis, to be published separately, indicated that the intervention was highly cost effective.

\section{Discussion}

This study, to our knowledge, is the first to assess the effect of systematic ascertainment of smoking and default delivery of evidence based cessation support by hospital based cessation practitioners. Our findings demonstrate a doubling of validated cessation rates at four weeks after discharge and highly significant improvements in service uptake when evidence based smoking interventions ${ }^{4}$ are delivered by default to all smokers by specialist staff. This study differs from previous research into smoking cessation in hospital settings, which has typically focused on the content of interventions rather than on means of optimising service provision and uptake.

\section{Strengths and limitations of the study}

The statistical significance of our primary outcome comparison of validated cessation at four weeks may have been compromised by the effect of clinical opposition to cessation intervention on oncology wards. However, the magnitude of the differences in service uptake, and significance of the primary outcome difference in a retrospective analysis that excluded patients with cancer, indicate strongly that this approach to smoking management in secondary care has the potential to increase sustained cessation after hospital admission. In view of the large numbers of smokers admitted to secondary care, the public health impact of wider implementation of this approach is potentially massive.

We carried out our study in a single centre for logistical and cost reasons, although the magnitude of intervention effects may differ between secondary care settings in relation to the level of intervention in current usual care. However, the finding that provision of cessation support by default improves service uptake and cessation rates will probably generalise to any centre not currently offering default intervention. A recent systematic review suggests that this is the case in most centres. ${ }^{17}$ Default service provision by properly trained staff, rather than reliance on existing staff to tackle smoking as part of their routine clinical work, also overcomes staff reluctance to intervene, low confidence, low prioritisation, lack of skills, and other barriers to smoking intervention in practice. ${ }^{9}{ }^{12} 18-24$ Current UK practice recommended by Department of Health, which relies on existing staff to ascertain smoking and refer smokers to local stop smoking services for further cessation support, ${ }^{25}$ has been shown to result in low uptake of such community services after discharge. ${ }^{26}$ But initiating behavioural support at the bedside of patients, as in our study, largely prevented this attrition, resulting in a substantially increased service uptake. We were unable to include all smokers admitted to study wards, including many whose admissions began and ended outside the weekday working hours in which the service was available, and those unwilling to consent to provide outcome measures for the study. Further work is therefore needed to develop effective methods to capture these groups.

Our use of cluster randomisation by ward, which we adopted to prevent contamination of intervention and usual care groups, precluded us from exploring differential intervention effects within wards or medical specialties, since all patients on the respiratory ward were randomised to usual care and all patients on the cardiac ward randomised to the intervention. However, published evidence suggests that admission diagnosis is not a major determinant of the likelihood of cessation ${ }^{6}$. We used a 10 ppm threshold of exhaled carbon monoxide to define non-smoking, in line with the Russell standard, ${ }^{15}$ although all but one non-smoker had values below $8 \mathrm{ppm}$. There was no allocation concealment in this study, because wards (clusters) were randomised before patients were recruited. However, because all clusters were randomised at the same time and patients are admitted to wards according to sex and specialty, selection bias in relation to intervention is unlikely.

Participants were all provided with the same information about the trial, which did not include specific information about the different interventions offered. Provision of information about smoking cessation support and the request for follow-up information may have increased demand for and uptake of usual care support. But any such bias would have reduced the difference in treatment effects, and any reporting bias would have been mitigated by the use of biochemical validation techniques. Exhaled carbon monoxide only assessed short term abstinence from smoking, but we chose this measure because other measures involving cotinine were inappropriate, in view of the potential for continued use of dual nicotine replacement therapy at follow-up data collection.

The longer median length of stay in the intervention group meant that smokers in this group had to remain abstinent for longer that patients in the usual care group, although a median difference of one day seems unlikely to account for a doubling in smoking cessation. We were unable to ascertain adherence to dual nicotine replacement therapy, but it is likely that measures to increase both uptake and adherence would have further improved the cessation rate. There is no reason to believe 
that adherence differed between the two study groups and thus have any influence on the results reported. Despite being offered dual nicotine replacement therapy every day throughout admission, most patients in the intervention group accepted or received only one session. This finding indicated that the promising results demonstrated by this trial arose predominantly from the delivery of one behavioural intervention, often but not invariably in conjunction with dual nicotine replacement therapy. Therefore, the delivery of behavioural support early in the admission was probably a key determinant of cessation success.

\section{Sensitivity analysis}

The intervention group's doubled rate of validated cessation at four weeks after discharge was of borderline significance. Our sensitivity analysis indicated that this finding was partly due to the low and biased recruitment of patients from oncology wards, but also arose from a higher degree of clustering within wards than expected when the study was planned (intraclass correlation coefficient 0.07 rather than 0.02), and low numbers of participants $(\mathrm{n}<10)$ on four wards. The results are, however, promising in terms of service uptake and potential for validated smoking cessation. Although the study design precluded blinding, ward staff were unaware of the exact details of the study, and patients were specifically not informed of the components of the intervention being tested. Consent was not required for any aspect of the intervention delivery because it formed part of what should be standard NHS practice; instead, consent was only sought for the follow-up measures, for which the procedure was identical for both groups. It is therefore unlikely that knowledge of missing out on treatment influenced the results gained in the study.

Previous studies of smoking cessation interventions in hospitals have tended to focus on the content of the intervention, aiming to improve effectiveness, rather than developing the mode of delivery to improve reach. Our intervention combined components that had been previously shown to be effective ${ }^{6}$ but with no new or experimental content; the observed improved efficacy was generated purely by ensuring more comprehensive coverage. Our findings thus complement assessments of the "Ottawa Model" approach. This approach draws on front line medical staff to assess smoking status, deliver smoking cessation support, and ensure follow-up after discharge using an interactive telephone system that is mediated by voice response. The Ottawa Model improves reach but to a lesser extent than in the present study. ${ }^{27}$

Improvements in smoking ascertainment were also reported in another study of a systematic approach, involving an "improvement team" of physicians, nurses, and the medical director in partnership with the New Hampshire tobacco prevention and control programme. The study aimed to increase referral and use of the state sponsored "Try To Stop Tobacco Resource Centre," but did not report the quit rates achieved. ${ }^{28}$ The importance of continuity in providing support for smoking that transcends the interface between inpatient and community care was highlighted in a UK study by Lewis and colleagues. ${ }^{29}$ They found that uptake of community based support was more likely if it was organised before discharge within one week of transfer from hospital services.

\section{Clinical implications and conclusions}

Delivery and uptake of smoking cessation treatment, and hence quit rates, can be improved substantially in smokers admitted to secondary care wards by using simple measures to ensure systematic ascertainment of smoking, to deliver treatment, and to ensure community follow-up for smokers willing to accept help. Further research is necessary to optimise the design and efficiency of this service provision model for inpatients, and to adapt the service for non-medical specialties. However, the institutionalised delivery of simple interventions early in the hospital stay, if widely adopted, could make a substantial contribution to individual and population health.

Contributors: RLM is the guarantor and takes responsibility for the integrity of the work as a whole, from inception to publication; contributed to the study conception and design, acquisition of data, interpretation of data, drafting the article, and approved the final version of the manuscript. JL-B analysed the data, contributed to the drafting of the article, interpretation of data, and approved the final version of the manuscript. JM and LJ contributed to the acquisition of data and approved the final version of the manuscript. SP contributed to the study conception and design, drafting the article, and approved the final version of the manuscript. JL contributed to interpretation of the data, drafting the article, and approved the final version of the manuscript. JB contributed to the study conception and design, interpretation of data, drafting the article, and approved the final version of the manuscript.

Funding: This paper presents independent research funded by the National Institute for Health Research under its Programme Grants for Applied Research programme (RP-PG-0608-10020). The views expressed in this presentation are those of the authors and not necessarily those of the NHS, the National Institute for Health Research, or the Department of Health.

Competing interests: All authors have completed the ICMJE uniform disclosure form at www.icmje.org/coi_disclosure.pdf and declare: no support from any organisation for the submitted work; no financial relationships with any organisations that might have an interest in the submitted work in the previous three years; no other relationships or activities that could appear to have influenced the submitted work.

Ethical approval: Ethical approval for the study was provided by the Nottingham research ethics committee 1 (ref 10/H0403/34).

Patient consent: All participants gave informed consent before taking part in the study.

Data sharing: No additional data available

Doll R, Peto R, Boreham J, Sutherland I. Mortality in relation to smoking: 50 years observations on male British doctors. BMJ 2004:328:1519.

2 Parrott S, Godfrey C, Raw M, West R, McNeill A. Guidance for commissioners on the cost effectiveness of smoking cessation interventions. Thorax 1998;53(supp 5):AS1-38. Tengs TO, Adams ME, Pliskin JS, Safran DG, Siegel JE, Weinstein MC, et al. 500 Lifesaving interventions and their cost-effectiveness. Risk Analysis 1995;15:369-90. National Institute for Health and Clinical Excellence. Brief interventions and referral for smoking cessation in primary care and other settings. NICE, 2006.

5 US Department of Health and Human Services Public Health Service. Treating tobacco use and dependence: 2008 update. May 2008. www.ahrq.gov/professionals/cliniciansproviders/guidelines-recommendations/tobacco/clinicians/treating_tobacco_use08.pdf.

6 Rigotti NA, Clair C, Munafo MR, Stead LF. Interventions for smoking cessation in hospitalised patients. Cochrane Database Syst Rev 2012;5:CD001837.

7 Fiore MC, Bailey WC, Cohen SJ, et al. Treating tobacco use and dependence. clinical practice guideline. Department of Health and Human Services.Public Health Service, 1996.

8 Raw M, McNeill A, West R. Smoking cessation guidelines for health professionals-a guide to effective smoking cessation interventions for the health care system. Thorax 1998;53:S1-19.

9 Goldstein MG. Missed opportunities to assist hospitalized smokers. Am J Prev Med 1999;17:317-8.

10 Hajek P, Taylor TZ, Mills P. Brief intervention during hospital admission to help patients to give up smoking after myocardial infarction and bypass surgery: randomised controlled trial. BMJ 2002;324:87-9.

11 Murray R, Leonardi-Bee J, Marsh J, Jayes L, Britton J. Smoking status ascertainment and interventions in acute medical patients. Clin Med 2012;12:59-62.

12 Vaughn TE, Ward MM, Doebbeling BN, Uden-Holman T, Clarke WT, Woolson RF. Organizational and provider characteristics fostering smoking cessation practice guideline adherence: an empirical look. J Ambul Care Manage 2002;25:17-31.

13 National Health Service. SmokeFree. 2013. http://smokefree.nhs.uk/resources/.

14 British Heart Foundation. Stop smoking: how to quit for a healthy heart. 2010. www.npt. gov.uk/PDF/BHF_Stop_Smoking_guide.pdf.

15 West R, Hajek P, Stead L, Stapleton J. Outcome criteria in smoking cessation trials: proposal for a common standard. Addiction 2005;100:299-303.

16 Molyneux A, Lewis S, Leivers U, Anderton A, Antoniak M, Brackenridge A, et al. Clinical trial comparing nicotine replacement therapy (NRT) plus brief counselling, brief counselling 


\section{What is already known on this topic}

Ascertaining smoking status in patients and providing cessation support for smokers willing to accept it, has been recommended in clinical guidelines in the US and UK for over 15 years

But implementation of this guidance in secondary care remains far from complete

High intensity, behavioural interventions initiated in hospital and including support for at least one month after discharge have been shown to increase smoking cessation among patients in hospital, regardless of admitting diagnosis

\section{What this study adds}

This trial reports the effect of a systematic intervention to identify smokers and offer bedside support by specialist staff, ensuring community follow-up for smokers willing to accept help

Increases in quit rates (validated by measuring exhaled carbon monoxide) at four weeks, smoking cessation delivery, and service uptake demonstrate that simple measures could, if widely adopted, make a substantial contribution to individual and population health

alone, and minimal intervention on smoking cessation in hospital inpatients. Thorax 2003;58:484-8.

17 Freund M, Campbell E, Paul C, McElduff P, Walsh RA, Sakrouge R, et al. Smoking care provision in hospitals: a review of prevalence. Nicotine Tob Res 2008;10:757-74.

18 Hawkshaw BA, Zuo Y. Audit of prescribed nicotine replacement therapy to hospital inpatients who smoke. Med J Aus 2005;182:43-4.

19 May FC, Stocks N, Barton C. Identification of barriers that impede the implementation of nicotine replacement therapy in the acute cardiac care setting. Eur $\mathrm{J}$ Cardiovasc Prev Rehabil 2008;15:646-50.

20 Rigotti NA, Arnsten JH, McKool KM, Wood-Reid KM, Singer DE, Pasternak RC. The use of nicotine-replacement therapy by hospitalized smokers. Am J Prev Med 1999:17:255-9.

21 Thy T, Boker T, Gallefoss F, Bakke PS. Hospital doctors' attitudes toward giving their patients smoking cessation help. Clin Respir J 2007;1:30-6.

22 Vega S, Stolare I. Smoking cessation education increases interventions in a New Zealand hospital: World No Tobacco Day revisited. N Z Med J 2010;123:35-40.

23 Warner DO, Sarr MG, Offord KP, Dale LC. Anesthesiologists, general surgeons, and tobacco interventions in the perioperative period. Anesth Analg 2004;99:1766-73.

24 Warner KE. The framework convention on tobacco control: opportunities and issues. Salud Publica Mex 2008;50 (suppl 3):S283-91.

25 Department of Health. NHS stop smoking services: service and monitoring guidance-2011/12. Department of Health, 2011.
26 National Centre for Smoking Cessation and Training. Streamlined secondary care system project report. 2012. www.ncsct.co.uk/usr/pub/ncsct-streamlined-secondary-care-finalreport.pdf.

27 Reid RD, Mullen KA, Slovinec D'Angelo ME, Aitken DA, Papadakis S, Haley PM, et al. Smoking cessation for hospitalized smokers: an evaluation of the "Ottawa Model". Nicotine Tob Res 2010;12:11-8.

28 Liu SK, Prior E, Warren C, Brown T, Snide J, Butterly JR. Improving the quality of care for the hospitalized tobacco user-one institution's transformational journey. J Cancer Educ 2010;25:297-301.

29 Lewis KE, Durgan L, Edwards VM, Dixon H, Whitehead C, Sykes RN. Can smokers switch from a hospital-based to a community-based stop smoking service? An open-label, randomized trial comparing three referral schemes. Nicotine Tob Res 2009;11:756-64.

\section{Accepted: 14 June 2013}

\section{Cite this as: BMJ 2013;347:f4004}

This is an Open Access article distributed in accordance with the Creative Commons Attribution Non Commercial (CC BY-NC 3.0) license, which permits others to distribute, remix, adapt, build upon this work non-commercially, and license their derivative works on different terms, provided the original work is properly cited and the use is non-commercial. See: http://creativecommons.org/licenses/by-nc/3.0/. 


\section{Tables}

Table 1| Demographic data of study participants

\begin{tabular}{|c|c|c|c|}
\hline & Usual care $(n=229)$ & Intervention $(n=264)$ & $\mathbf{P}^{*}$ \\
\hline Age (years, mean (SD)) & $57.9(15.2)$ & $55.0(13.3)$ & 0.028 \\
\hline Male sex (no (\%) of participants) & $120(52)$ & $176(67)$ & 0.001 \\
\hline \multicolumn{4}{|c|}{ Discharge rate of ward (no (\%) of participants) } \\
\hline Low rate & $38(17)$ & $61(23)$ & \multirow[t]{2}{*}{0.072} \\
\hline High rate & $191(83)$ & $203(77)$ & \\
\hline \multicolumn{4}{|c|}{ Specialty of ward (no of participants (no of wards)) } \\
\hline Stroke & $60(3)$ & $11(1)$ & \multirow[t]{8}{*}{$<0.00$} \\
\hline Oncology & $10(1)$ & $35(3)$ & \\
\hline Renal & 0 & $42(2)$ & \\
\hline Cardiac & 0 & $151(1)$ & \\
\hline Infectious disease & 0 & $18(1)$ & \\
\hline Diabetes & $13(1)$ & $7(1)$ & \\
\hline Blood or haematology & $17(2)$ & 0 & \\
\hline Respiratory & $129(2)$ & 0 & \\
\hline
\end{tabular}

$\mathrm{SD}=$ standard deviation

*Two sided $\mathrm{P}$ value relates to two sample $t$ test for continuous variables and $\mathrm{x}^{2}$ test for categorical variables. 


\begin{tabular}{|c|c|c|c|c|}
\hline & Usual care (\%) & Intervention (\%) & Adjusted odds ratio $(95 \% \mathrm{Cl})^{*}$ & $\mathbf{P}$ \\
\hline \multicolumn{5}{|l|}{ Primary outcome } \\
\hline $\begin{array}{l}\text { Quit smoking at } 4 \text { weeks (continuous } \\
\text { abstinence), CO validated } \dagger\end{array}$ & $17(n=37)$ & $38(n=98)$ & 2.10 (0.96 to 4.61$)$ & 0.06 \\
\hline \multicolumn{5}{|l|}{ Secondary outcomes } \\
\hline $\begin{array}{l}\text { Offered behavioural support or } \\
\text { pharmacotherapy (or both) as an inpatient }\end{array}$ & $46(n=106)$ & $100(n=264)$ & NA & - \\
\hline Accepted behavioural support as an inpatient§ & $29(n=17)$ & $70(n=185)$ & 5.71 (3.58 to 9.09$)$ ฯ & $<0.001$ \\
\hline $\begin{array}{l}\text { Abstained from smoking at discharge, } \mathrm{CO} \\
\text { validated }\end{array}$ & $29(n=67)$ & $58(n=151)$ & $1.95(0.94$ to 4.05$)$ & 0.07 \\
\hline $\begin{array}{l}\text { Discharged with any pharmacotherapy for } \\
\text { smoking cessation }\end{array}$ & $27(n=62)$ & $49(n=128)$ & 3.95 (1.81 to 8.63$)$ & $<0.001$ \\
\hline $\begin{array}{l}\text { Referred to a stop smoking service after } \\
\text { discharge }^{\star \star}\end{array}$ & $6(n=13)$ & $55(n=144)$ & $21.8(9.4$ to 50.6$)$ & $<0.001$ \\
\hline $\begin{array}{l}\text { Received support from a stop smoking service } \\
\text { after discharge }{ }^{\star \star}\end{array}$ & $10(n=21)$ & $31(n=80)$ & $4.22(2.27$ to 7.83$) \dagger \dagger$ & $<0.001$ \\
\hline $\begin{array}{l}\text { Quit smoking between discharge and } 6 \text { month } \\
\text { follow-up (continuous abstinence), CO } \\
\text { validatedł‡ }\end{array}$ & $9(n=19)$ & $19(n=47)$ & $1.53(0.60$ to 3.91$)$ & 0.37 \\
\hline
\end{tabular}

All analyses based on nine clusters in each treatment group. $\mathrm{NA}=$ not applicable; $\mathrm{CO}$ validated=cessation validated by measuring levels of exhaled carbon monoxide. ${ }^{*}$ Adjusted for cluster design of the trial and for the stratification variable (high $v$ low discharge rate).

†Usual care, $n=224$; intervention, $n=260$.

fUsual care, $n=229$; intervention, $n=264$.

§Usual care, $n=58$; intervention, $n=264$.

ILogistic regression model with adjustment for stratification variable allowing for the cluster design of the trial using robust standard errors.

**Usual care $n=229$; intervention $n=262$.

††Model excluded the stratification variable as random effect not estimable.

¥łUsual care, $n=219$; intervention, $n=250$. 


\section{Figure}

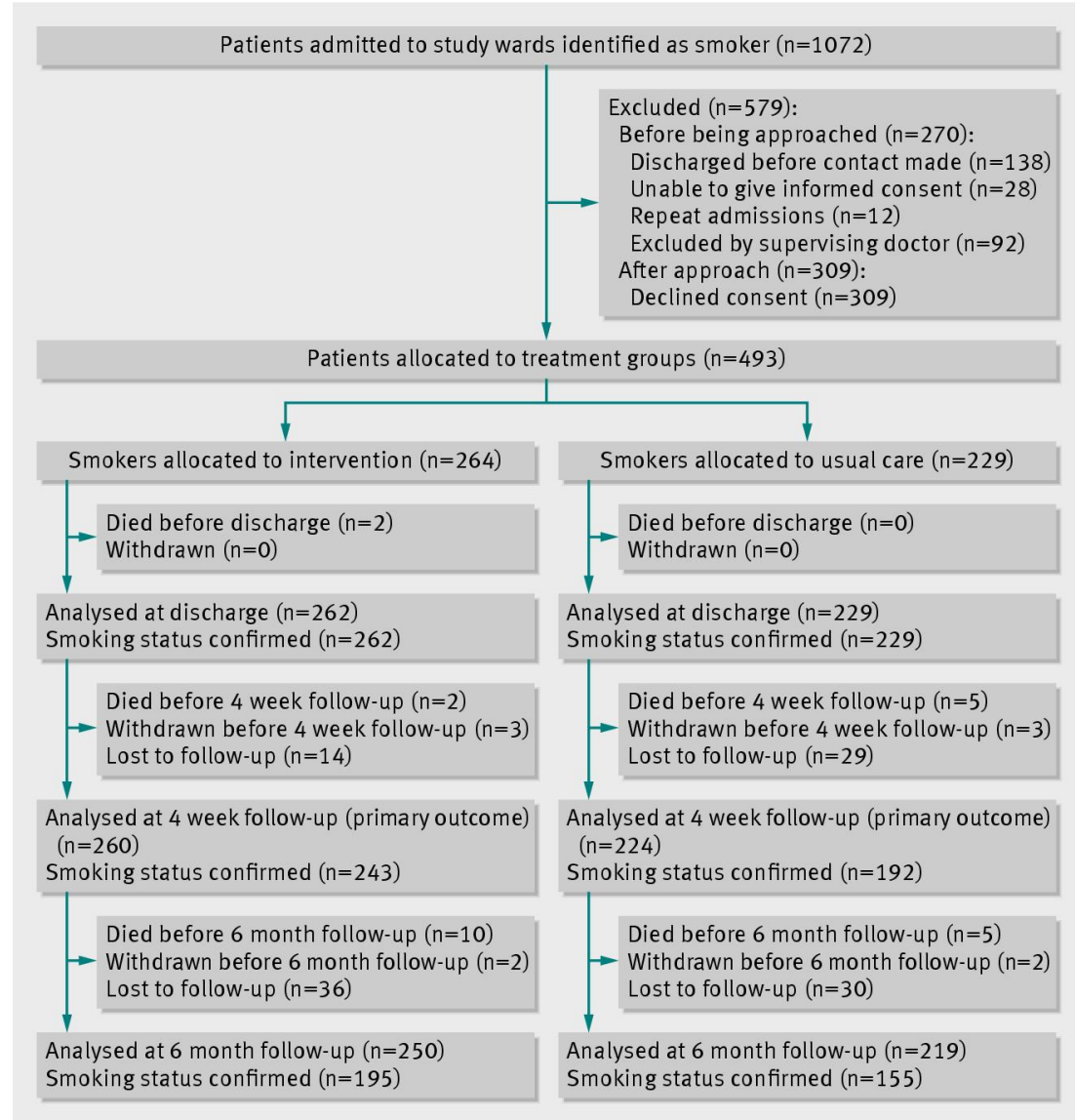

Flow of study participants 